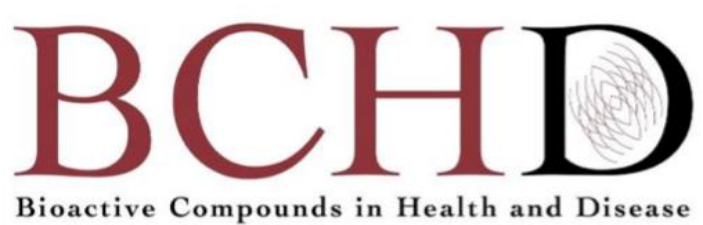

\title{
Call for mobilization of functional foods, antioxidants, and herbal antivirals in support of international campaign to control coronavirus
}

\author{
Reza Ratmanesh ${ }^{1}$, Francesco Marotta ${ }^{1}$, Ishak Tekin ${ }^{2}$ \\ ${ }^{1}$ Re-Genera R\&D International Research group for Aging Intervention and San Babila Clinic, Milan, Italy; ${ }^{2 Z o n g u l d a k}$ Bulent \\ Ecevit University, School of Medicine, Department of Immunology, Zonguldak, Turkey \\ Corresponding Author: Reza Rastmansh; ReGenera R\&DInternational for Aging Intervention, Milan, Italy
}

Submission Date: March 28 ${ }^{\text {th }}, 2020 ;$ Acceptance Date: May 28 ${ }^{\text {th }}, 2020$; Publication Date: May 31 2020

Please Cite This Article As: Ratmanesh R., Marotta, Tekin I. Call for mobilization of functional foods, antioxidants, and herbal antivirals in support of international campaign to control coronavirus. Bioactive Compounds in Health and Disease. 2020; 3(5):90-95. DOI: https:/doi.org/10.31989/bchd.v3i5.717

\section{ABSTRACT}

Objective: The unavailability of optimal medication and shortage of effective vaccines for COVID-19 may offer an opportunity for alternative natural therapies. We suggest re-visiting the hidden potential of traditional remedies including functional foods, antioxidant, and antiviral herbs. We suggest clinical trials investigating antioxidant and/or pre- pro-biotic supplements in patients with COVID-19 infections and the measurement of appropriate variables such as ROS, RNS, IgA, SOD, lysozyme production, RIBs, and food intake to yield preliminary data for future trials. Data from current patients and victims could be re-analyzed retrospectively in an attempt to understand the effects on the immune system, herbal consumption behavior, and routine nutritional measures.

Keywords: Functional foods, antioxidant, antiviral, coronavirus, COVID-19

CFFC 2020. This is an Open Access article distributed under the terms of the Creative Commons Attribution 4.0 License (http://creativecommons.org/licenses/by/4.0).

\section{INTRODUCTION}

Novel coronavirus from patients with pneumonia in China is likely to be caused by 2019-nCoV that falls into the genus betacoronavirus. This genus includes coronaviruses (SARS-CoV, bat SARS-like CoV, and others) discovered in humans, bats, and other wild animals [1]. Coronaviruses are a group of viruses that cause diseases in mammals and birds [2]. In humans, the viruses cause respiratory infections, like the common cold, which are typically mild $[3,4]$.

Along with current international campaigns for designing a vaccine for the coronavirus to cure or prevent cases at the individual level, it will be an influential policy to use traditional alternative medicine practices. These include using traditional remedies paired with simple methods like hand hygiene [5], mouth rinse, and nasal spray with povidone-iodine mouthwash [6]. It is, however, interesting that in a systematic review, Singh et al [7] found studies showing some improvement in the prevention and treatment of upper respiratory tract infections. They postulate that hypertonic saline gargles and nasal wash may be useful in prevention and for the care of patients with COVID-19. Their evidence emphasizes the need for randomized controlled trials to evaluate the role and mechanism 
of nasopharyngeal wash in COVID-19 [7]. There are still no published reports on the potential of antiviral herbs when used for_gargling or mouth washing. However, there are some reports that functional foods [8, 9], antioxidants such as vitamin C $[10,11]$, and vitamin $\mathrm{D}$ may be effective in preventing primary and secondary infections from coronavirus [12, 13].

The unavailability of optimal medication and the shortage of effective vaccines may offer an opportunity for the utilization of alternative natural therapies [14]. Natural antiviral and antibacterial herbs give a limitless chance for future clinical trials. Several herbal remedies have already been used for prevention and treatment of viral respiratory illnesses as indicated by Mousa [14]. Among those that were found to be effective are maoto, licorice roots, antiwei, North American ginseng, berries, Echinacea, plant extracted carnosic acid, pomegranate, guava tea, and Bai Shao.

\section{DISCUSSION}

Unfortunately, despite the long history of traditional usage and antiviral benefits of some herbs through in vitro studies, there is currently no clinical evidence to prove their beneficial effects in respiratory viral infections, which warrants future clinical trials. For example, red bryony is traditionally used for relief from colds and flu [15-17]. At least two ribosomeinactivating proteins (RIBs) have been isolated and characterized from the roots of Bryonia dioica during in vitro studies. One of these was a novel 27 kDa protein termed bryodin 2 (BD2), while the second was a previously reported RIP, referred to here as bryodin 1 (BD1) [18].

There is no report on the potential efficacy of BD2 and BD1 in the inhibition or reduction of replication and morphogenesis of new Coronavirus or any kind of virus in clinical settings as of yet. However, what shed some promising light on the potential of future clinical trials is that some herbs such as red bryony have high potency to inactivate different types of ribosomes [18-22], which may show broadspectrum antiviral activity, something of interest due to its potentially to partially control viruses, including coronavirus.

As another example, it has been shown that some natural extracts from Chinese herbs that have traditionally been used for the treatment and prevention of epidemic diseases, such as pseudolaric acid $B$, oridonin, and erianin, by inducing $\mathrm{G} 2 / \mathrm{M}$ phase arrest, might be good candidates for inhibiting human enterovirus 68 production [23]. There are other mounting in vitro studies supporting the antiinfluenza effects of natural herbs with different mechanisms. These mechanisms include NF-KB and JAK-STAT signaling pathways [24], the induction of type I interferon-related signaling and the antiviral state in HEp2 cells [25], the inhibition of the proliferation of influenza viruses of various strain in vitro (with the $50 \%$ inhibitory concentration and blocking the early stages (0-2 h) of virus infection), the suppression of virus-induced NF-kB activation, the alleviated virus-induced gene expression of IL-6, IL-8, TNF-a, IP-10, and monocyte chemoattractant protein-1 in a dose-dependent manner [26], just to mention a few of many in vivo and in vitro studies.

Obviously, examples are not just limited to antiviral herbs and the same story applies to the use of prebiotics, probiotics, natural antivirals, antioxidants, and functional foods in clinical settings.

In the hopes of protecting mankind from airborne transmitted viral diseases, oxidants play a robust role in the metabolic regulation of the host and viral replication signaling pathways. Oda et al. [27] reported that administration of pyran polymer conjugated superoxide dismutase would protect mice challenged with lethal doses of influenza virus. Indeed, the extracellular reactive oxygen species (ROS) and reactive nitrogen species (RNS) have a pathogenetic impact in influenza-induced acute lung injury, since lungs have several potential cellular sources of ROS and the upregulated xanthine oxidase contributes to further enhancement of the oxidative stress response [28, 29].

Following the observation that antioxidant-rich diets may be of some generic benefit in this clinical setting [30], several herbal extracts have more specifically tested in vitro against viral [14] early this year [31]. But so far, no clinical data has been convincingly attempted. In 2000 at UC Berkley, it was found that a GMP/ISO Asian fermented papaya preparation (FPP-ORI) could protect from DNA and protein damage [32] and stimulate IFN-alpha (24). Several other papers confirmed its redox- and immune-modulator properties, as confirmed by Professor Montagnier, a Nobel laureate for HIV discovery (25).

Thus, in 2012, Marotta et al. ran a randomized, cross-over, placebo-controlled clinical study administering FPP-ORI 9g/day to 90 GSTM1-positive sedentary healthy patients divided into three groups: A) 20-40 years of age; B) 41-65 years of age; C) over 65 years of age. FPP-ORI significantly increased salivary secretion rate in the youngest age-group and a significantly higher level of $\lg A$ and lysozyme production, irrespective of age, while their baseline production was remarkably lower in the oldest agegroup. Moreover, FPP-ORI treatment enabled a significant upregulation of all phase II enzyme and superoxide dismutase gene (SOD) (i.e. the most important antioxidant in the respiratory tract) expression tested in nasal lavage cells [35]. The 
biological significance of these effects remains to be demonstrated in longer clinical trials, but may already offer a promising avenue to pursue.

Needless to say, it is an emergency and there is no extra time to run clinical trials to assess the exact antiviral "efficacy" or wait for release of results and publications. Considering the fact that there have been no serious side effects to natural herbs, prebiotics, probiotics, antioxidants, or functional foods in common colds and flu, if used properly, it seems a wise measure to consider such a potential at least to prevent secondary respiratory infections (either bacterial or viral) in household and public places. This can be done by ventilating the rooms, using gargles prepared from natural antiviral herbs, and consuming prebiotic and probiotic enrich products and antioxidants.

We previously presented a hypothesis on the potential of prebiotics and probiotics to enhance the efficacy of HIV vaccination earlier, in which we theorized that artificial over-expression of heat shock proteins might enhance efficacy of HIV vaccination in humans. There is only one case report of a patient who developed bacteraemia and septic pulmonary emboli with Lactobacillus acidophilus after taking a probiotic containing this organism [36], however, S. boulardii has been used to treat 33 HIV patients with chronic diarrhea. In these doubleblind studies, 56\% of patients receiving $\mathrm{S}$. boulardii had resolution of diarrhea compared with only $9 \%$ of patients receiving placebo. There were no cases of bacteraemia or septic pulmonary emboli in either study [37, 38]. Overall, such studies will also give encouragement to industries to think about possibility of pre/ pro-biotic formulations for a higher efficacy of HIV vaccination worldwide.

Severe 2019-nCoV infection cases have these symptoms: acute respiratory distress syndrome, septic shock, metabolic acidosis, bleeding, and coagulation dysfunction. Furthermore, like SARS-CoV and MERS-CoV, 2019-nCoV infections induce production of high levels of cytokines [39]. In 2003, glucocorticoid was widely used in SARS treatment to control pulmonary infection by regulating inflammatory responses. The inflammatory response of the body also plays a crucial role in SARS-induced lung injury cases. It is important to control the mass production of cytokines and inflammatory response, given that they are responsible for the accumulation of cells and fluids in CoV pneumonia [40]. At this point, we need a strategy for regulating the immune response and controlling excessive inflammation without compromising the beneficial host defense. Coagulopathy and thromboembolism are proving to be prevalent in severe COVID-19 and are related to decreased survival. Coagulation is an intricate balance between clot promoting and dissolving processes in which vitamin $\mathrm{K}$ (as a nutraceutical) plays a well-known role [41].

Phytochemicals, especially polyphenols have important anti-inflammatory effects by regulating innate and adaptive immunity through the modulation of different cytokines. They have the ability to scavenge free radicals and inactivate other pro-oxidants, but they also have anti-inflammatory behaviors which inhibit the activation of NF-KB, inducible nitric oxide synthases, pro-inflammatory enzymes such as cyclooxygenase-2, mitogenactivated protein kinases, and protein kinase-C. The majority of herbal phenolic compounds alter signaling and enzymatic processes involved in inflammation such as tyrosine and serine-threonine protein kinases. They modulate inflammatory mediators such as cytokines, peptides, and arachidonic acid metabolites [42].

Excessive inflammation may account for the severity of the disease in extreme cases. This inflammation may lead to pulmonary embolism, requiring alternative treatments other than just the standard ventilation treatment. A number of antiinflammatory plants are known and very recently have been systematically reviewed by Zhang and Lis [43], such as Opuntia species [44] and bryonia [18, 22]. Additionally, Thabti et al. recently analyzed the antiviral properties of the leaves and stem bark of the mulberry tree (Morus spp.) in vitro. They compared the antiviral activity of Morus spp. on enveloped and nonenveloped viral pathogens, such as human coronavirus (HCoV 229E) and different members of the Picornaviridae family-human poliovirus 1, human parechovirus 1 and 3 , and human echovirus 11 . The antiviral activity of 12 water and water-alcohol plant extracts of the leaves and stem bark of three different species of mulberry-Morus alba var. alba, Morus alba var. rosa, and Morus rubra-were evaluated. They also evaluated the antiviral activities of kuwanon G against HCoV-229E. These evaluations showed that several extracts reduced the viral titer and cytopathogenic effects (CPE). Leaves' water-alcohol extracts exhibited maximum antiviral activity on human coronavirus, while stem bark and leaves' water and water-alcohol extracts were the most effective on picornaviruses [45].

\section{CONCLUSION}

Threats should always be treated as an opportunity and although it is a painful lesson, we suggest that clinical trials investigating antioxidant and/or prepro-biotic supplements in patients with respiratory infections are conducted and appropriate variables such as ROS, RNS, IgA, SOD, lysozyme production, RIBs, and food intake are measured. This information 
can then be used as preliminary data for future trials. We also suggest that data from current patients and victims be re-analyzed retrospectively in an attempt to find out any effect from immune system, herbal consumption behavior, and routine nutritional measures. The lesson from in vitro studies about the ribosome-inactivating property of some natural products as well as antioxidant/immune-stimulating functional supplements that act locally within the oral cavity as shown for FPP-ORI [46], may open a new veil of investigation of viral infections, bacterial infections and viral-bacterial co-infections in the respiratory tract in future clinical trials. While we all try to understand the mechanism of infection and outline definitive therapy for COVID-19, considering the recurrent nature of COVID-19, it is necessary to take every useful measure to control such global pandemics.

LIST OF ABBREVIATIONS: COVID-19: Coronavirus 2019, RIB: ribosome-inactivating proteins, BD: bryodin, NF-kB: nuclear factor kappa B, SOD: superoxide dismutase, JAK/STAT: Janus Kinase/Signal Transducer and Activator of Transcription, IL: Interleukin, IFN-alpha: interferon-alpha, ROS: reactive oxygen species, RNS: reactive nitrogen species, HIV: human immunodeficiency viruses, IgA: Immunoglobulin A.

ACKNOWLEDGMENTS AND FUNDING: Authors received no funding for this paper.

COMPETING INTERESTS: Authors have no competing interest.

AUTHORS' CONTRIBUTIONS: Authors have equally contributed to this paper.

\section{REFERENCES:}

1. Tan W, Zhao X, Ma X, Wang W, Niu P, Xu W. A novel coronavirus genome identified in a cluster of pneumonia casesG_łWuhan, China 2019Gê 2020. China CDC Weekly 2020; 2(4):61-62.

2. Chan JF-W, To KK-W, Tse H, Jin DY, Yuen KY. Interspecies transmission and emergence of novel viruses: lessons from bats and birds. Trends in Microbiology 2013; 21(10):544-555.

3. Berry M, Gamieldien J, Fielding BC. Identification of new respiratory viruses in the new millennium. Viruses 2015; 7(3):996-1019.

4. Pyrc K, Berkhout B, van der Hoek L. Identification of new human coronaviruses. Expert Review of Anti-infective Therapy 2007; 5(2):245-253.

5. World Health Organization. Rational use of personal protective equipment for coronavirus disease (COVID-19): interim guidance, 27 February 2020. In: World Health Organization; 2020.

6. Kirk-Bayley J, Challacombe S, Sunkaraneni S, Combes J. The Use of Povidone lodine Nasal Spray and Mouthwash During the Current COVID-19 Pandemic May Protect Healthcare
Workers and Reduce Cross Infection. Available at SSRN 35630922020.

7. Singh S, Sharma N, Singh U, Singh T, Mangal DK, Singh V. Nasopharyngeal wash in preventing and treating upper respiratory tract infections: Could it prevent COVID-19? Lung India 2020; 37(3):246.

8. Mustafa MZ, Shamsuddin SH, Sulaiman SA, Abdullah JM. Antiinflammatory Properties of Stingless Bee Honey May Reduce the Severity of Pulmonary Manifestations in COVID-19 Infections. Malaysian Journal of Medical Sciences 2020; 27(2).

9. Dhar D, Mohanty A. Gut microbiota and Covid-19-possible link and implications. Virus Research 2020:198018.

10. Cheng RZ. Can early and high intravenous dose of vitamin C prevent and treat coronavirus disease 2019 (COVID-19)? Medicine in Drug Discovery 2020; 5:100028.

11. Wang JZ, Zhang RY, Bai J. An anti-oxidative therapy for ameliorating cardiac injuries of critically ill COVID-19-infected patients. International journal of cardiology 2020.

12. Grant WB, Lahore H, McDonnell SL, Baggerly CA, French CB, Aliano JL, et al. Evidence that vitamin $D$ supplementation could reduce risk of influenza and COVID-19 infections and deaths. Nutrients 2020; 12(4):988.

13. Grant WB, Lahore H, McDonnell SL, Baggerly CA, French CB, Aliano JL, et al. Vitamin D supplementation could prevent and treat influenza, coronavirus, and pneumonia infections. 2020.

14. Mousa HA. Prevention and Treatment of Influenza, InfluenzaLike Illness, and Common Cold by Herbal, Complementary, and Natural Therapies. J Evid Based Complementary Altern Med 2017; 22(1):166-174.

15. Andrea H.Zengion, EricYarnell. Herbal and Nutritional Supplements for Painful Conditions. In: Pain Procedures in Clinical Practice; 2011. pp. 178-204.

16. Pankaj Pradhan, Neha Kaushik, Pankaj Bhateja, Abhimanyu. REMEDIAL APPROACH: SWINE FLU. International Journal of Pharmaceutical Sciences Review and Research 2010; 4(2):74.

17. Rigat M, Vall_ذs J, IgI_رsias J, Garnatje T. Traditional and alternative natural therapeutic products used in the treatment of respiratory tract infectious diseases in the eastern Catalan Pyrenees (Iberian Peninsula). Journal of Ethnopharmacology 2013; 148(2):411-422.

18. Siegall CB, Gawlak SL, Chace D, Wolff EA, Mixan B, Marquardt $\mathrm{H}$. Characterization of ribosome-inactivating proteins isolated from Bryonia dioica and their utility as carcinoma-reactive immunoconjugates. Bioconjug Chem 1994; 5(5):423-429.

19. Bolognesi A, Barbieri L, Abbondanza A, Falasca Al, Carnicelli $D$, Battelli MG, et al. Purification and properties of new ribosome-inactivating proteins with RNA N-glycosidase activity. Biochim Biophys Acta 1990; 1087(3):293-302

20. Francisco JA, Gawlak SL, Miller M, Bathe J, Russell D, Chace D, et al. Expression and characterization of bryodin 1 and $a$ bryodin 1-based single-chain immunotoxin from tobacco cell culture. Bioconjug Chem 1997; 8(5):708-713.

21. Srivastava S, Verma HN, Srivastava A, Prasad V. BDP-30, a systemic resistance inducer from Boerhaavia diffusa L., suppresses TMV infection, and displays homology with ribosome-inactivating proteins. J Biosci 2015; 40(1):125-135.

22. Stirpe F, Barbieri L, Battelli MG, Falasca Al, Abbondanza A, Lorenzoni $\mathrm{E}$, et al. Bryodin, a ribosome-inactivating protein from the roots of Bryonia dioica L. (white bryony). Biochem J 1986; 240(3):659-665.

23. Meng $X, Y u X$, Liu C, Wang Y, Song F, Huan C, et al. Effect of ingredients from Chinese herbs on enterovirus D68 production. Phytother Res 2019; 33(1):174-186.

24. Ding Y, Chen L, Wu W, Yang J, Yang Z, Liu S. Andrographolide inhibits influenza $A$ virus-induced inflammation in a murine model through NF-kappaB and JAK-STAT signaling pathway. Microbes Infect 2017; 19(12):605-615.

25. Lee $B H$, Chathuranga $K$, Uddin MB, Weeratunga P, Kim MS, Cho WK, et al. Coptidis Rhizoma extract inhibits replication of 
respiratory syncytial virus in vitro and in vivo by inducing antiviral state. J Microbiol 2017; 55(6):488-498.

26. Ding Y, Zeng L, Li R, Chen $Q$, Zhou B, Chen $Q$, et al. The Chinese prescription lianhuaqingwen capsule exerts anti-influenza activity through the inhibition of viral propagation and impacts immune function. BMC Complement Altern Med 2017; 17(1):130.

27. Oda $T$, Akaike $T$, Hamamoto $T$, Suzuki F, Hirano $T$, Maeda $H$. Oxygen radicals in influenza-induced pathogenesis and treatment with pyran polymer-conjugated SOD. Science 1989; 244(4907):974-976.

28. Akaike $\mathrm{T}$, Ando $\mathrm{M}$, Oda $\mathrm{T}$, Doi $\mathrm{T}$, ljiri $\mathrm{S}$, Araki $\mathrm{S}$, et al. Dependence on 02 - generation by xanthine oxidase of pathogenesis of influenza virus infection in mice. $J$ Clin Invest 1990; 85(3):739-745.

29. Hennet T, Peterhans E, Stocker R. Alterations in antioxidant defences in lung and liver of mice infected with influenza $A$ virus. J Gen Virol 1992; 73 ( Pt 1):39-46.

30. Han SN, Meydani M, Wu D, Bender BS, Smith DE, Vina J, et al. Effect of long-term dietary antioxidant supplementation on influenza virus infection. J Gerontol A Biol Sci Med Sci 2000; 55(10):B496-B503.

31. Yan W, Chen J, Wei Z, Wang X, Zeng Z, Tembo D, et al. Effect of eleutheroside $B 1$ on noncoding RNAs and protein profiles of influenza A virusinfected A549 cells. Int J Mol Med 2020.

32. Rimbach G, Guo Q, Akiyama T, Matsugo S, Moini H, Virgili F, et al. Ferric nitrilotriacetate induced DNA and protein damage: inhibitory effect of a fermented papaya preparation. Anticancer Res 2000; 20(5A):2907-2914.

33. Aruoma OI, Hayashi Y, Marotta F, Mantello P, Rachmilewitz E, Montagnier L. Applications and bioefficacy of the functional food supplement fermented papaya preparation. Toxicology 2010; 278(1):6-16.

34. Mankowski RT, Leeuwenburgh C, Manini TM, Woods AJ, Anton SD. Effects of Fermented Papaya Preparation (FPP) on Safety Outcomes in Older Adults - A Short Report of a Placebo-Controlled Clinical Trial. J Frailty Aging 2018; 7(2):142-146.

35. Marotta F, Naito Y, Jain S, Lorenzetti A, Soresi V, Kumari A, et al. Is there a potential application of a fermented nutraceutical in acute respiratory illnesses? An in-vivo placebo-controlled, cross-over clinical study in different age groups of healthy subjects. J Biol Regul Homeost Agents 2012; 26(2):285-294.

36. Reza Rastmanesh, Franceco Marotta. Potential of Prebiotics and Probiotics to Enhance the Efficacy of HIV Vaccination: A Hypothesis. Metabolomics 2012; 2(1).

37. Born P, Lersch C, Zimmerhackl B, Classen M. [The Saccharomyces boulardii therapy of HIV-associated diarrhea]. Dtsch Med Wochenschr 1993; 118(20):765.

38. Saint-Marc T, Rossello-Prats L, Touraine JL. [Efficacy of Saccharomyces boulardii in the treatment of diarrhea in AIDS]. Ann Med Interne (Paris) 1991; 142(1):64-65.

39. Li JY, You Z, Wang $Q$, Zhou ZJ, Qiu Y, Luo R, et al. The epidemic of 2019-novel-coronavirus (2019-nCoV) pneumonia and insights for emerging infectious diseases in the future. Microbes Infect 2020.

40. Channappanavar R, Perlman S. Pathogenic human coronavirus infections: causes and consequences of cytokine storm and immunopathology. Semin Immunopathol 2017; 39(5):529-539.

41. Dofferhoff AS, Piscaer I, Schurgers L, Walk J, Van Den Ouweland JM, Hackeng TM, et al. Reduced Vitamin K Status as A Potentially Modifiable Prognostic Risk Factor in COVID19. 2020.

42. Ishak ضzel Tekin, Francesco Marotta. Polyphenols.Academic Press Prevention and Treatment of Human Disease. In: Polyphenols and Immune System; 2018. pp. 236-276.

43. Zhang L, Liu Y. Potential interventions for novel coronavirus in China: A systematic review. Journal of medical virology 2020; 92(5):479-490.

44. Park Ds, Ahn M, Go GM. Functional bioactivity of Opuntia species. Oriental Pharmacy and Experimental Medicine 2004; 4(4):219-226.

45. Thabti İ, Albert Q, Philippot Sر, Dupire Fد, Westerhuis B, Fontanay $S$, et al. Advances on Antiviral Activity of Morus spp. Plant Extracts: Human Coronavirus and Virus-Related Respiratory Tract Infections in the Spotlight. Molecules 2020; 25(8):1876.

46. Fibach E, Ginsburg I. The antioxidant effect of fermented papaya preparation in the oral cavity. Phytotherapy Research 2015; 29(9):1317-1322. 